\title{
Les Établissements Fouga de Béziers (1920-1966)
}

The Fouga Establishments at Béziers (1920-1966)

\section{Raoul Balso}

\section{OpenEdition}

\section{Journals}

Édition électronique

URL : https://journals.openedition.org/rhcf/1753

DOI : 10.4000/rhcf.1753

Éditeur

Rails \& histoire

Édition imprimée

Date de publication : 1 décembre 2003

ISBN : 0996-9403

ISSN : 0996-9403

Référence électronique

Raoul Balso, "Les Établissements Fouga de Béziers (1920-1966)", Revue d'histoire des chemins de fer [En ligne], 28-29 | 2003, mis en ligne le 19 décembre 2014, consulté le 22 avril 2022. URL : http:// journals.openedition.org/rhcf/1753; DOI : https://doi.org/10.4000/rhcf.1753

Ce document a été généré automatiquement le 22 avril 2022

Tous droits réservés 


\title{
Les Établissements Fouga de Béziers (1920-1966)
}

\author{
The Fouga Establishments at Béziers (1920-1966)
}

\author{
Raoul Balso
}

1 La société Fouga prend en 1920 la suite des ateliers de la Compagnie du Midi. Les usines, installées à Béziers dans la plaine Saint-Pierre, jouxtent les installations de la gare : elles emploient de 1800 à 2200 ouvriers et constituent le plus gros noyau industriel de la ville. Le professeur Édith Claustre écrit : "Grâce aux établissements Fouga, l'image économique de Béziers au début du XXe siècle se résumait en deux thèmes : viticulture et métallurgie du rail.»

2 Aujourd'hui, les établissements Fouga dont les derniers ateliers ont fermé en 1966 ne sont déjà plus qu'un souvenir et il est difficile de retracer leur histoire. L'essentiel des sources a été rassemblé par un ancien ouvrier, Georges Martzel, membre du syndicat CGT des métaux, qui les a présentées dans quatre plaquettes d'une centaine de pages.

3 Dans le cadre de ce colloque nous nous sommes concentrés sur les usines de réparation de matériel ferroviaire de Béziers et ne faisons que seulement citer l'usine d'aviation d'Aire-sur-Adour qui a sorti le Fouga Magister 170-R.

4 Après avoir présenté les établissements Fouga (implantation, usines, conseil d'administration et personnel) nous avons suivi leur évolution à travers le Front populaire (les grèves) la guerre (avec le bombardement du 4 juillet 1944) et l'aprèsguerre (conflits sociaux) jusqu'à leur liquidation fin 1966. Leur reconversion dans des domaines autres que ferroviaire n'a pas réussi.

\section{9-1924 - Implantation dans la plaine Saint-Pierre}

5 Les Établissements Fouga et Cie ont été créés le 6 décembre 1919 sous la forme d'une société anonyme au capital de 5 millions de francs, ayant son siège social à Béziers, plaine Saint-Pierre. Leur but est d'assurer la réparation des wagons de marchandises de tous types pour le compte de la Compagnie du Midi. Cette dernière, dans le contexte 
social et économique de l'immédiat après-guerre - plus de 7000 grévistes sont dénombrés dans le Biterrois de 1920 -, décide de fermer ses ateliers et licencie son personnel.

Les Établissements Fouga s'installent sur un terrain vague planté en vignes situé dans la plaine Saint-Pierre, au sud-est de Béziers, entre les voies de triage du Capiscol et le canal du Midi. Ce terrain de $42500 \mathrm{~m} 2$ a été loué en l'état à la nouvelle société, à charge pour elle d'y créer des ateliers de wagonnage (fig. 1).

Figure 1. Localisation des usines Fouga dans Béziers : au pied de la ville, dans la zone ferroviaire. Cadenat fr.,Béziers, 1932.

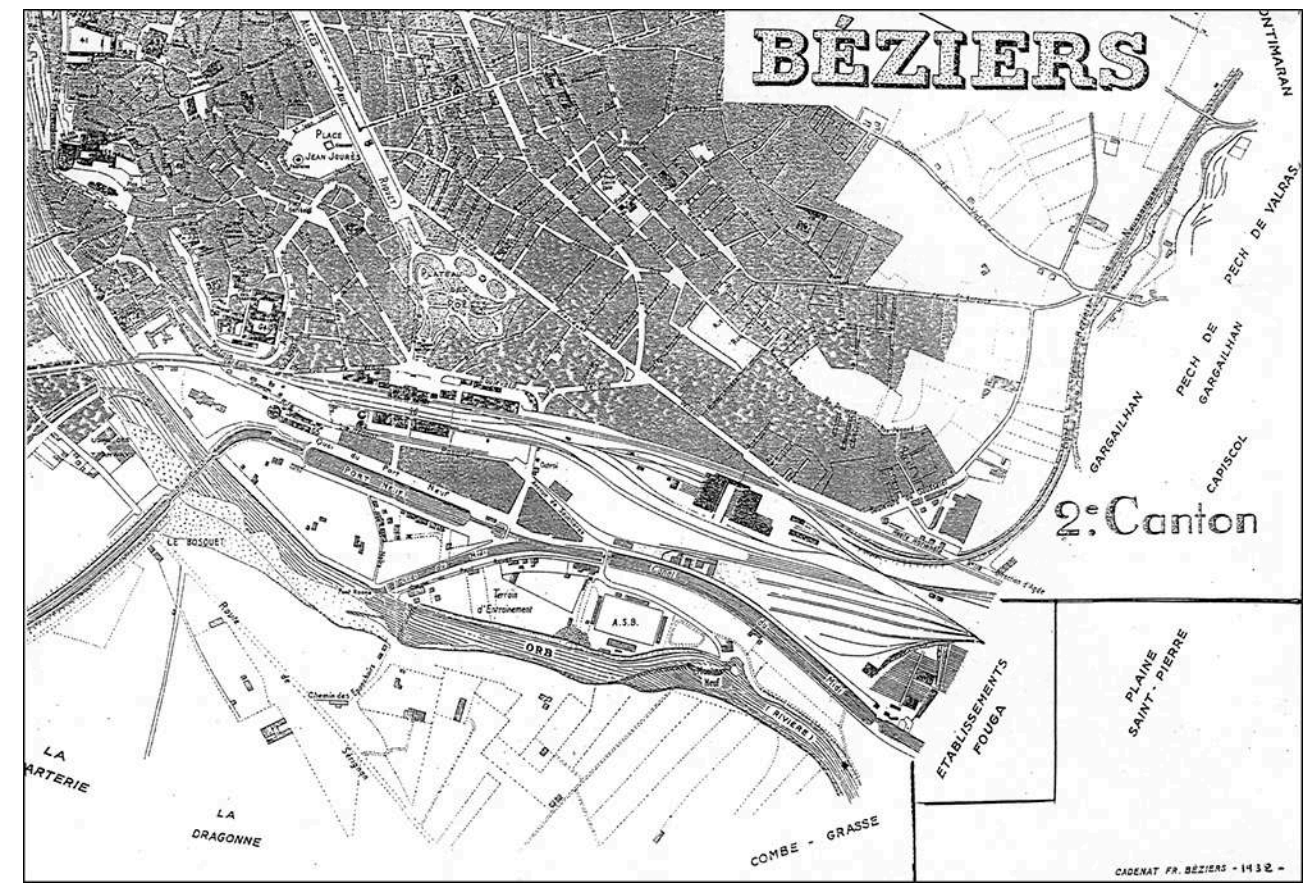

COLL. PART., CL. D.R.

7 La nouvelle usine, étudiée, aménagée et mise en exploitation par Gaston Fouga, ancien ingénieur de la Compagnie du Midi, obtient de nombreux contrats pour la réparations de wagons et réalise des bénéfices substantiels qui permettent aux Établissements Fouga de développer leurs activités : ils achètent un terrain de $91200 \mathrm{~m} 2$ en bordure du canal du Midi et, de 1920 à 1923, construisent de vastes ateliers pour la réparation des locomotives, des voitures de $2 \mathrm{e}$ et $3 \mathrm{e}$ classes, des voitures-restaurants et des voitureslits de la Compagnie internationale des wagons-lits.

L'ensemble - ateliers nord wagons et ateliers sud voitures - relié au réseau ferroviaire par un embranchement particulier, est complété par les magasins centraux d'approvisionnement et par tous les services du siège social : comptabilité générale, études, achats, contentieux, bureaux techniques. Trois augmentations de capital traduisent cette expansion : le capital social passe des 5 millions de 1919 à 10 millions de francs en mars 1920, 15 millions de francs en avril 1921 et 20 millions de francs en octobre 1924. La majorité des actions-bons de cinq cents francs $7 \%$ au porteur (fig. 2) est placée dans la bourgeoisie biterroise. 
Figure 2. 1922. Bon de $500 \mathrm{~F} / 7 \%$ au porteur émis à Béziers.

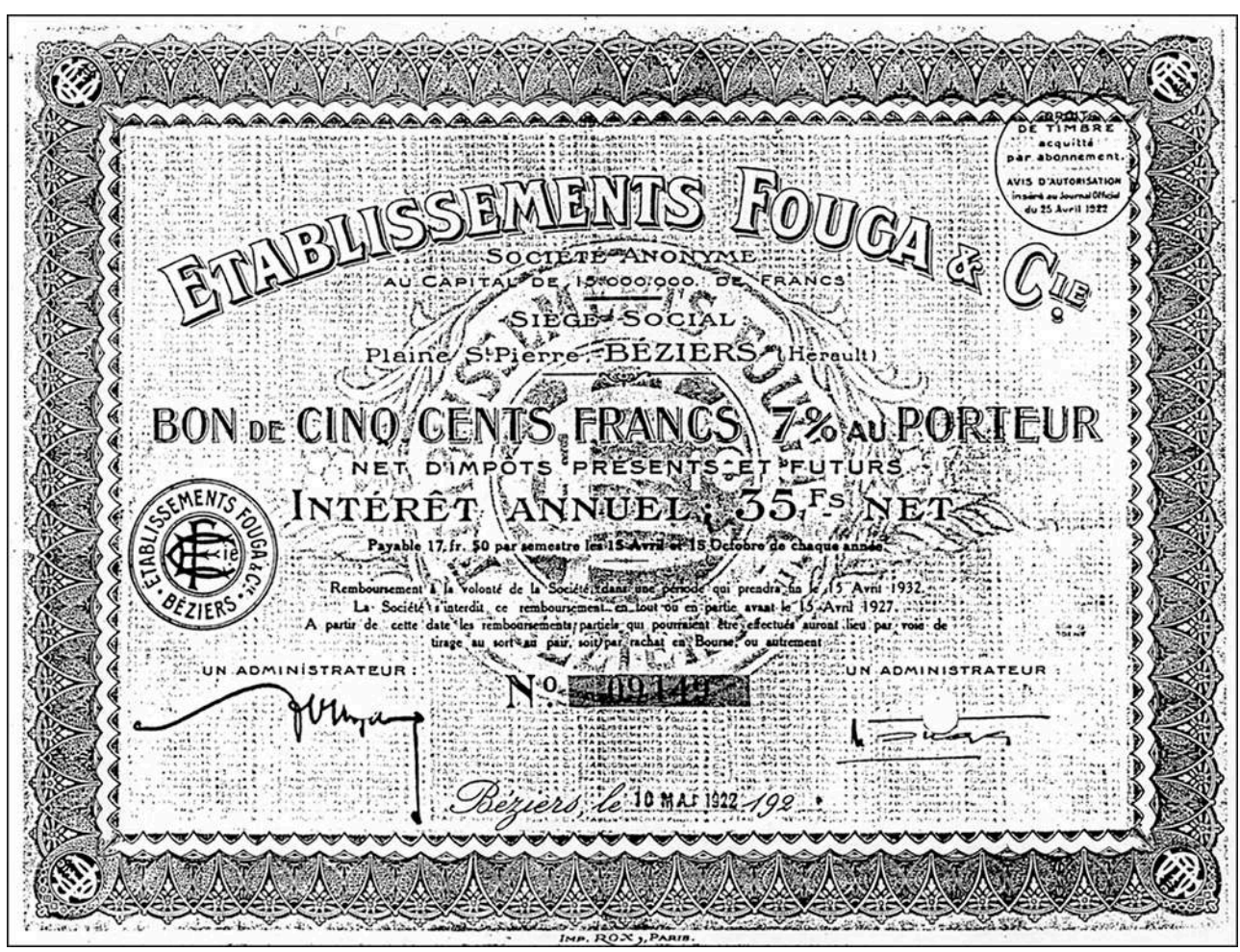

COLL. PART., CL.D.R.

\section{Importance des établissements ferroviaires : ateliers nord et Fouga sud}

9 Les ateliers nord et sud, leurs bâtiments, leurs moyens de manutention leur outillage, leur organisation, sont, au départ, strictement conçus et orientés vers la réparation du matériel roulant du chemin de fer.

10 Ateliers de réparation de wagons (surface couverte $7000 \mathrm{~m}^{2}$ - surface non couverte $35000 \mathrm{~m} 2$ - total $42000 \mathrm{~m} 2$ ). Ces ateliers comportent des voies principales d'entrée et de sortie, des voies transversales qui desservent le hall des grandes réparations, le chantier des moyennes réparations, le chantier de levage ainsi que le parc des wagons en attente de réparation et les parcs à pièces et à essieux. Toutes ces voies, d'une longueur totale de $10200 \mathrm{~m}$, sont desservies par des chariots transbordeurs électriques à niveau.

11 Ateliers de réparation de voitures (surface couverte $8000 \mathrm{~m} 2$ - surface non couverte $7000 \mathrm{~m}^{2}$ - total $15000 \mathrm{~m}^{2}$ ). Ces ateliers comprennent deux halles de $10 \mathrm{~m}$ de large sur $100 \mathrm{~m}$ de long desservies par deux ponts roulants de 8 tonnes. Les voies placées en travers de l'atelier sont desservies par un chariot transbordeur à fosse de 75 tonnes et 18 mètres. Autour de lui sont groupées les sections serrurerie, menuiserie, tôlerie et zinguerie.

12 Ateliers de réparation de locomotives (surface couverte $19000 \mathrm{~m}^{2}$-surface non couverte $32000 \mathrm{~m}^{2}$ - total $51000 \mathrm{~m}^{2}$ ). Ces ateliers rassemblent : 
13 17 mètres. Pour faciliter les opérations, il dispose d'un pont roulant de 50 tonnes (déboîtage des chaudières et mises sur roues) de 4 vérins électriques de 100 tonnes (grosses pièces) et de deux ponts roulants de 8 tonnes (petites manutentions) ; - sept ateliers spécialisés traitent la grosse chaudronnerie, les essais de freins, les soudures autogènes, le régulage, les essais de moteurs, les roues, les ressorts à lame. d'électricité et d'une forge complètent ce complexe. "Les établissements Fouga constituent une unité parfaite dont les services sont parfaitement imbriqués entre eux et d'un fonctionnement harmonieux favorisé par la présence de ces trois branches d'activité sous une seule direction.»

\section{Gaston Fouga et le conseil d'administration} membres, compte surtout des ingénieurs et des magistrats consulaires. président de chambre au tribunal de commerce de la Seine, juge honoraire à ce même tribunal et Gaston Fouga, ingénieur à la Compagnie du Midi. Finances et Henri Bouvier, ancien élève de l'École polytechnique. Le directeur général est Henri Froustey, ingénieur des Arts et Métiers, ingénieur à la Compagnie du Midi. Robert Froustey fils, ingénieur des Arts et Manufactures, Raymond Kourilsky, ancien élève de l'École polytechnique, Jules Rienne, ancien président de chambre au tribunal de la Seine, Pierre Goar, ingénieur du Crédit foncier d'Algérie et de Tunisie, administrateur des établissements Rouge à Narbonne, Robert de Chassey, administrateur des distilleries Bresson à Béziers, Jacques Valette, ingénieur des Arts et Métiers, ingénieur à la Compagnie du Midi.

21 Plusieurs sont unis par des liens familiaux : Jacques Sicre est le beau-père de Gaston Fouga, Robert Froustey est le fils d'Henri Froustey, Raymond Kourilsky est le gendre de Léon Nadau.

Gaston Fouga, assisté de ses anciens collègues, Froustey et Valette, assure la liaison effective des établissements. Grand (1,85 m), puissant, compétent, il agit en patron absolu: sa politique, bien que paternaliste, se heurte souvent à la résistance du personnel.

\section{Les Fougassiens : ouvriers, employés, apprentis}

Assez rapidement, le nombre total du personnel (cadres, employés, ouvriers) avoisine 1500 personnes, hommes, femmes et jeunes confondus.

24 Ces salariés, de catégories très diverses, viennent d'un peu partout. On y trouve des ouvriers de la Compagnie du Midi, licenciés en 1920, des métallurgistes venus de toute 
la France (Paris, le Nord, le Sud, l'Est), des Italiens chassés de leur pays par le fascisme et aussi des ouvriers agricoles attirés par des salaires nettement supérieurs à ceux du vignoble.

Beaucoup d'élèves de l'École pratique de Béziers munis d'un brevet deviennent des ouvriers hautement qualifiés, chefs d'équipe, contre maîtres.

Les ateliers étant situés à trois ou quatre kilomètres de Béziers, les ouvriers et les employés se rendent à leur travail à bicyclette. Chaque jour, ce sont des cohortes de bicyclettes qui arrivent de Béziers et aussi des localités voisines : Nissan, Bassan, Valras, Sérignan. Les horaires sont ponctués par une puissante sirène qui rappelle la discipline qui régit le travail ; rendement aux «boni » avec des temps alloués qui imposent un rendement maximum. Dans l'ensemble, on travaille aux pièces neuf heures par jour et six jours par semaine.

« À la gare on est fonctionnaire; aux Docks méridionaux on est employé ; il n'y a qu'à Fouga qu'on est vraiment des ouvriers. » Effectivement, les Fougassiens ont l'air d'être faits sur le même modèle, avec la même mentalité et la même manière de vivre. Au fil des ans, par le biais des "délégués d'ateliers", puis des représentants des syndicats, ils vont affirmer leur solidarité et marquer la politique locale. «Fouga, là-bas au bord du canal, dans un lieu un peu excentré, c'est une sorte de poudrière, une sorte de volcan, volcan qui, de temps en temps, lâche des masses de gens qui viennent manifester et qui montent en ville. » (Marie Rouanet, romancière.)

\section{5 - L'usine d'Aire-sur-Adour : du planeur à l'avion Fouga Magister 170-R}

En 1935, Gaston Fouga, qui s'intéresse à l'aviation, rachète une entreprise aéronautique en difficulté à Aire-sur-Adour et installe des ateliers qui se spécialisent dans la réparation et la production de différents types de planeurs.

Le département aviation de Fouga (surface couverte $11800 \mathrm{~m}^{2}$ - surface non couverte $36400 \mathrm{~m} 2$ - total $58200 \mathrm{~m} 2$ ) s'adjoindra des ateliers de montage et d'essais en vol d'avions à Toulouse-Blagnac (surface couverte $6500 \mathrm{~m}^{2}$ - surface non couverte 23000 $\mathrm{m}^{2}$ - total $39500 \mathrm{~m}^{2}$ ) et, tout en restant intégré à la société mère, fera une brillante carrière.

En 1949, après la guerre, les ateliers d'Aire-sur-Adour sortent le planeur de performance C.M.8.15, puis le premier Magister 170-R qui décolle le 23 juillet 1952 sur la piste de Mont-de-Marsan. En 1954, le département aviation de la société Fouga reçoit du ministère de la Défense la commande d'une série de 100 appareils Fouga dits Magister 170-R.

En 1956, la société Fouga étant mise en règlement judiciaire, son département aviation est cédé aux sociétés Potez-Fouga (union financière de cinq sociétés). En mai 1958, la gestion générale est reprise par le groupe Potez. Potez Air Fouga équipe l'École de l'air de Salon-de-Provence, la patrouille de France et fournit des Magister 170-R à de nombreux pays jusqu'à son absorption par la SNIAS en janvier 1970. 


\section{Le Front populaire et les grandes grèves de 1936, 1937 et 1938}

31 Alors que le Front populaire triomphe en France, le congrès d'unité qui se réunit à Toulouse donne naissance à la CGT réunifiée et permet au Syndicat des métaux de renforcer son influence sur les établissements Fouga et sur la politique municipale. Joseph Lazare, Gaston Fabre, Charles Gibert, Marcel Néranson, René Pallot, Guy Bousquet sont élus sur les listes communistes; Jules Faigt et Étienne Bénéfice sur les listes socialistes. Joseph Lazare sera maire et Jules Faigt sénateur.

On trouve des Fougassiens dans les différentes associations de la ville, sportives, caritatives, parents d'élèves, mutuelles, office public d'HLM, clinique mutualiste. Ils participent à tous les grands conflits politiques et animent les grandes grèves de cette époque.

Début juin 1936, l'assemblée générale du Syndicat des Métaux, réunie à la maison du peuple de Béziers, décide la grève avec occupation de l'usine. Déclenchée le 10 juin, elle fut totale. Une collecte de vivres et d'argent est organisée pour le soutien aux grévistes auprès des commerçants, des jardiniers et de la population biterroise. La grève se termina dans la joie avec l'augmentation des salaires, la signature d'une convention collective et 15 jours de congés payés pour tous. Le 14 juillet il y a des bals dans tous les quartiers.

34 Le 14 mai 1937, la direction de Fouga décide de licencier six employés. L'action du personnel à l'appel du syndicat et l'intervention de M. Piquemal, chef adjoint du cabinet du ministre du Travail, amènent la direction à réintégrer les six employés dans leurs anciennes fonctions. L'état de conciliation est signé par l'administrateur Chasles.

À l'arrière-plan se profile la prochaine création de la Société nationale des chemins de fer français qui, le 31 août 1937, réunira toutes les compagnies déficitaires : le lien qui unissait la société Fouga à la Compagnie du Midi s'affaiblira.

Le 3 juin 1938, la direction de Fouga revient sur une partie des avantages accordés en 1936. Le 30 juin, le personnel se met en grève et occupe l'usine. Le 1er juillet la direction licencie tout le personnel. Le personnel, consulté sous la présidence des parlementaires locaux, se prononce pour la grève. Les résultats ( 1509 oui - 27 non et 21 nuls) sont communiqués à la presse et, malgré les menaces, l'occupation de l'usine continue. Le 1er août, les ateliers sont réquisitionnés par l'autorité militaire et, après plusieurs "sentences arbitrales », sont rendus à la société Fouga le 28 août. Dans la ville, le climat social reste particulièrement tendu.

Le 30 novembre 1938, un nouveau conflit aboutit au licenciement de 61 ouvriers (sur les 1700 qui n'avaient pas travaillé). Dans le contexte international de l'époque (guerre civile espagnole, politique expansionniste d'Hitler et de Mussolini) le patronat tente d'anéantir l'action syndicale.

Simultanément, le ministre des Travaux publics précise que le déficit de la SNCF ne sera pas couvert par l'État et que toutes ses grosses dépenses de réparation devront être ajournées.

La direction de Fouga, face aux réductions de ses contrats, annonce qu'elle va poursuivre sa politique de licenciement. Début 1939, elle demande le licenciement de 
500 ouvriers pour manque de commandes : la SNCF a réduit le volume des réparations de 25 à $35 \%$.

\section{9-1945 - La guerre : occupation, résistance et libération} juin, 35 métallos de Fouga sont arrêtés au cours d'une rafle monstre et internés dans le camp de Saint-Sulpice « comme individus dangereux à la sécurité de l'État. » Le 22 juin, l'armistice est signé à Rethondes.

En mars 1941, les ouvriers de Fouga sont appelés à défendre leurs salaires mais la misère s'installe avec les restrictions alimentaires et, bientôt, la peur. En avril 1941, une distribution de tracts demandant la libération des ouvriers emprisonnés entrâne l'arrestation de leurs épouses pour « menées antinationales ».

En 1942, malgré la répression, la Résistance s'organise et développe ses activités. En 1944, les sabotages se multiplient ; le 11 février, deux locomotives sautent à la sortie des ateliers; le 22 avril, un pylône électrique est dynamité... ${ }^{1}$ Peu avant la Libération, le 5 juillet 1944, les bombardiers alliés lâchent leurs bombes sur la gare de triage du Capiscol : les bâtiments de Fouga sont fortement endommagés.

Le 25 août 1944, dans une ville libérée, une délégation ouvrière prend contact avec la direction de Fouga et une convention collective provisoire est signée. Début septembre, un comité de Libération "épure " les directeurs et condamne les héritiers de Gaston Fouga à verser 10 millions de francs. Cette amende va permettre au comité de gestion d'animer les diverses commissions créées en faveur des ouvriers: ravitaillement, accidents du travail, sports, bibliothèque, colonie de vacances de Valras-Plage...

\section{5-1950 - Réduction des marchés SNCF - Recherche de reconversion}

Malgré la pénurie de matériaux et de machines-outils, la reconstruction des usines se poursuit. Le nouveau comité de gestion présidé par l'ingénieur Henry Froustey cherche à diversifier leurs productions : à côté de la réparation du matériel ferroviaire, la société se lance dans la fabrication de boulangeries de campagne pour l'armée, de tracteurs vignerons que recommande l'école d'agriculture de Montpellier et surtout du matériel pour les forages pétroliers. Pour financer ces programmes, le capital social passe de 20 à 40 millions de francs.

Le personnel, soutenu par le parti communiste (le député Lazare) et le parti socialiste (le député Faigt) manifeste cependant contre la guerre froide: grèves de juillet et décembre 1947. Comme le note le syndicaliste Martzel : « Les ouvriers s'intéressent aux événements politiques parce que la Société Fouga a pour principal client l'État qui, à travers les ministères des Finances, des Transports et de l'Air, fixe le volume des commandes réparations SNCF et aviation. »

En 1949, le plan Marshall ayant permis à la SNCF de moderniser son parc, le volume des réparations baisse et entraîne la disparition de 16 usines. En 1954, il ne restera plus que 22 ateliers de réparation (fig. 3). 
En 1950, Fouga arrache à la SNCF et à la CIWL deux contrats de 100000 heures mensuelles et obtient d'équiper les gares d'Hendaye et de Cerbère en appareils de levage destinés au transit des wagons spéciaux de la Société Transfesa. Fouga décide également de remplacer la fabrication des wagons foudre (bois) par des wagons citernes en métal, mais la situation du complexe reste précaire.

Figure 3. 1954. Liste des 22 ateliers de réparation de matériel ferroviaire titulaires d'un marché SNCF. La charge attribuée à Fouga est soulignée.

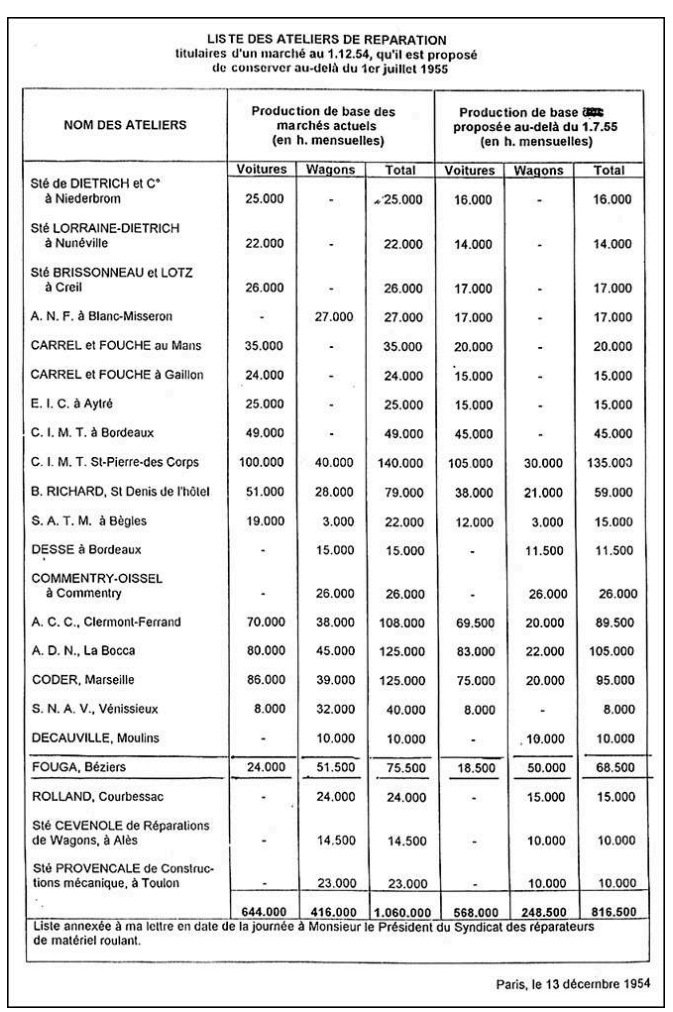

DOC. SNC. COLL. PART., CL. D.R.

\section{1-1966 - Conflits sociaux et fermeture progressive des usines}

Fin 1953, alors que les conflits se succèdent entre direction et personnel, le journal Le Monde (15 décembre) analyse la situation : «À Béziers, à côté de l'ancienne branche de réparation du matériel de Chemin de fer, l'activité comprend aujourd'hui la fabrication de grosses chaudronneries et de grosses mécaniques telles que pylônes de télécommunications, citernes, matériel pour raffineries de pétrole, mines de produits chimiques, centrales thermiques, équipements miniers. À Aire-sur-Adour, une autre usine avec bureau d'études de valeur à Toulouse, est spécialisée dans l'aviation légère. Grâce à elle, l'entreprise est au premier rang de l'aviation légère à réaction avec le Fouga 170-R dont une première commande de cent appareils vient d'être passée pour la formation et l'entraînement des pilotes de combat [...] Le chiffre d'affaire, passé de 981 millions en 1950 à 1257 millions en 1951, a atteint 1819 millions en 1952 à la suite de la reconversion de l'usine de Béziers qui intervient dans ce chiffre pour près de 50 \% [...] C'est pour mettre en harmonie le fond de roulement avec ce développement que cette 
société porte son capital de 120 millions à 240 millions de francs par l'émission au pair de quarante mille actions nouvelles de 3000 francs. Les souscriptions sont reçues dans les banques jusqu'à la fin de l'année. »

La situation continue cependant à se dégrader. En janvier 1955, le directeur administratif et le caissier principal sont arrêtés pour détournement de fonds (environ 13 millions de francs). En octobre, une nouvelle vague de licenciements donne lieu à des incidents graves : les directeurs sont maltraités par les ouvriers grévistes.

En avril 1958, la Société Fouga et Cie donne à bail à la Société Petro Fouga le fond industriel et commercial exploité dans l'usine sud. Le 28 juin 1958, la Société Fouga et Cie ferme son usine nord (fig. 4); 1200 ouvriers se trouvent sans travail. Sous la pression des syndicats le gouvernement désigne la Société Entreprise et Chantier pour rouvrir l'usine.

51 En 1961, Fouga et Cie vend les usines sud à la Société Petro Fouga (capital 3 millions de nouveaux francs) qui fait partie du groupe Commentry Oissel. Celui-ci, dirigé par trois conseillers de De Gaulle, François Lehideux, Georges Villiers président du Conseil du patronat français et Émile Roche, président du Conseil économique, refuse son soutien et, le 9 mars 1962, Petro Fouga dépose son bilan.

Figure 4. Juin 1958. Lettre notifiant la fermeture de l'usine Nord de Fouga. Cette lettre est intéressante par son en-tête : siège social à Paris - usines du groupe - liste des diverses activités.

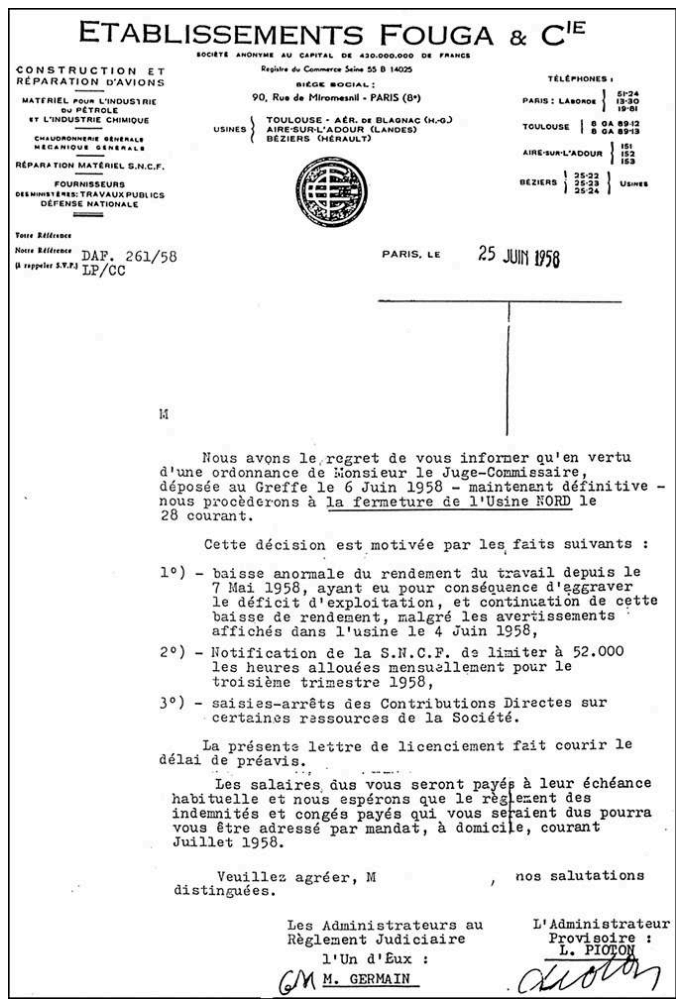

COLL. PART., CL. D.R.

Les ateliers sont alors bradés à divers repreneurs qui sont en fait des filiales de Fouga, comme la Compagnie européenne de matériel (CEMA) contrôlée par l'Intra Bank libanaise de Paris, la société Pantrama et la Société industrielle biterroise (SIB) : Du 15 novembre 1965 au 30 novembre 1966, cette dernière liquide définitivement les 
établissements Fouga. Il ne reste plus dans la plaine Saint-Pierre que les ateliers de la Cameron Iron Works du Texas qui fabriquent du matériel pétrolier.

Comme le souligne M. Tressol, président de la chambre de commerce, "L'arrêt de Fouga, c'est en deux ou trois ans, 1500 emplois de perdus, quelques trois ou quatre milliards de centimes de salaires qui sont versés en moins dans l'économie biterroise. » Une page de l'histoire locale est tournée.

\section{Conclusion}

\section{NOTES}

1. Voir par exemple l'action de Marcel Guy, syndicaliste, à l'usine puis après son entrée au maquis de Vabre où il est responsable du sabotage (site Internet de l'Amicale des maquis de Vabre, Maison de la montagne, 81330 Vabre : maquisdevabre.free.fr) (N.d.l.R.)

\section{RÉSUMÉS}

Nés après la Grande Guerre au bénéfice d'une étroite collaboration avec la Compagnie du Midi, les établissements Fouga ne sont plus aujourd'hui qu'un souvenir. Pour retracer leur histoire, qui a marqué Béziers et sa région jusqu'à la fermeture des derniers ateliers en 1966 et au-delà, on peut recourir à des sources heureusement rassemblées par un ancien ouvrier, Georges Martzel, membre du syndicat CGT des métaux. 
En abordant les grandes périodes de l'existence de l'entreprise, l'article centre son propos sur les usines de réparation de matériel ferroviaire de Béziers, l'usine d'aviation d'Aire-sur-Adour étant placée à la marge.

Après avoir présenté les établissements Fouga (implantation, usines, conseil d'administration et personnel) l'article suit leur évolution à travers le Front populaire (grèves, effacement de la Compagnie du Midi et création de la SNCF) et face à la contraction (dès la fin des années 1930 et jusque dans les années 1950) du marché réparation offert par la SNCF. La guerre est également abordée (bombardement du 4 juillet 1944) ainsi que l'après-guerre (conflits sociaux et tentatives de reconversion), jusqu'aux années conduisant à la liquidation en 1966.

The Fouga Establishments were born after the Great War, thanks to a close collaboration with the Compagnie du Midi. They are just a memory nowadays. To draw their history, which marked Béziers and its region until the closure of the last workshops in 1966 and beyond, one can to use the sources fortunately gathered by a former worker, Georges Martzel, a member of the CGT Metal Trade Union. In addressing the major periods of the existence of the Company, the author centers his comments on rail repair factories of Béziers (the aviation factory of Aire-sur-Adour being set apart).

After presenting the Fouga Company (implementation, factories, Board of Directors and staff) the article follows its evolution through the Popular Front (strikes, fading out of the Compagnie du Midi and creation of the SNCF) and the constant reduction of the repair contracts offered by SNCF (as early as the end of the 1930's and into the 1950's). The war is also discussed (bombing of the 4 th of July 1944) as well as post-war period (social conflicts, conversion), and the years leading to the liquidation in 1966.

\section{INDEX}

Mots-clés : atelier, entre-deux-guerres, industrie ferroviaire, stratégie d'entreprise, ville Keywords : business strategy, city, interwar period, railway industry, workshop

\section{AUTEUR}

\section{RAOUL BALSO}

Docteur en histoire, ingénieur honoraire de la SNCF 